Research Article

\title{
Combination of Red Clover and Hops Extract Improved Menopause Symptoms in an Ovariectomized Rat Model
}

\author{
Mi Ran Kim, Hyun Jin Kim, Su Hyun Yu, Bo Su Lee, Se Yeong Jeon, Jeong Jun Lee, \\ and Young Chul Lee 10
}

Natural Product Team, Naturech Co., Ltd., Chungcheongnam-do 31257, Republic of Korea

Correspondence should be addressed to Young Chul Lee; yclee@naturetech.co.kr

Received 21 January 2020; Revised 3 April 2020; Accepted 20 April 2020; Published 23 May 2020

Academic Editor: Yoshiki Mukudai

Copyright (C) 2020 Mi Ran Kim et al. This is an open access article distributed under the Creative Commons Attribution License, which permits unrestricted use, distribution, and reproduction in any medium, provided the original work is properly cited.

\begin{abstract}
Red clover and hops are already known for their alternative menopausal therapies; however, their combination has not yet been studied. This study aimed to evaluate the efficacy of the combination of red clover and hops extract (RHEC) for treating menopausal symptoms for the first time. A high-performance liquid chromatography (HPLC) method for RHEC was developed and validated for the analysis of biochanin $\mathrm{A}$ in red clover extract and xanthohumol in hops extract. An in vivo study was conducted using an ovariectomized rat model treated with RHEC $(125,250$, and $500 \mathrm{mg} / \mathrm{kg}$, p.o.) for a 12-week test period. Changes in body weight, tail skin temperature (TST), serum lipid profile, bone metabolism, antioxidants, and markers of vasorelaxation and uterus endometrium were evaluated. RHEC significantly inhibited body weight gain and decreased fat weight. Changes in TST associated with flashes were significantly inhibited in the RHEC groups. Other markers related to menopausal symptoms, such as blood lipid profile (total cholesterol and low-density-lipoprotein cholesterol), bone metabolism (serum alkaline phosphatase, osteocalcin, and c-terminal telopeptide type 1), antioxidants (superoxide dismutase and malondialdehyde), and vasorelaxants (endothelin-1 and nitric oxide), were significantly improved after the administration of RHEC. We also confirmed the safety of RHEC through histopathological observation of the endometrium. Our findings demonstrate that RHEC appears to have high potential for comprehensively improving various symptoms of menopause.
\end{abstract}

\section{Introduction}

Menopause is a natural process that develops in women typically in their late 40 s and involves a decrease in the estrogen levels, particularly due to ovarian decline $[1,2]$. This decrease in estrogen levels is accompanied by various symptoms, such as hot flashes, night sweats, and depression [3]. Estrogen deficiency can lead to an increased risk of metabolic diseases, such as obesity, heart disease, diabetes, and hypertension [4-6]. Furthermore, it affects the atherogenic lipid profile, such as the levels of high-density-lipoprotein (HDL) and low-density-lipoprotein (LDL) cholesterol [7].

Hormone therapy is the main treatment option for menopausal symptoms; however, long-term treatment via this approach can cause cardiovascular events and breast cancer [8]. Concerns about the safety of hormonal products have increased the popularity of many complementary therapies such as red clover extract. Various menopausal symptoms reduce the quality of life of menopausal women, so it is important to manage these symptoms healthily and properly through dietary supplements that lack side effects.

Red clover (Trifolium pratense L.) has estrogenic effects due to the presence of isoflavones, which are plant-based chemicals having estrogen-like effects on the body [9]. Thus, red clover botanical dietary supplements have been used for treating menopausal symptoms and for maintaining or improving cardiovascular health. The main isoflavones of red clover are biochanin $\mathrm{A}$, formononetin, ononin, and sisotrin. Upon ingesting red clover, biochanin A and formononetin are demethylated and metabolized to genistein and daidzein in the body [10].

Hops (Humulus lupulus L.) are the main ingredient used to brew beer and has been traditionally used in Europe to 
facilitate sleep and reduce mental stress. Hops contain phytochemicals with a wide range of biological activities, including chemopreventive, cardioprotective, and anti-inflammatory effects [11]. Hops dietary supplements have been studied as botanical alternatives to hormone therapy for relief from hot flashes and related symptoms in menopausal women [12]. The main components of hops are xanthohumol (chalcone), isoxanthohumol (flavone), and 8prenylnaringenin (prenylated flavonoid) [13, 14].

Based on the abovementioned information, we expect that the combination of red clover extract and hops extract (RHEC) can improve the various symptoms of the menopause. In this study, we investigate the effects of RHEC on tail skin temperature (TST), serum lipid profile, bone metabolism, antioxidant, and markers of vasorelaxation using an ovariectomized model (OVX) that displays a decline in estrogen levels similar to that in menopausal women.

\section{Materials and Methods}

2.1. RHEC Preparation. The red clover (leaf) and hops (flower and flower buds) used in the experiment were purchased from a herbal market (Danyang, China). The method of extraction was as follows: red clover $(200 \mathrm{~g})$ was extracted three times with $70 \%$ ethanol $\left(85^{\circ} \mathrm{C}\right.$ and $3 \mathrm{~h}$ reflux). The extract was filtered through a filter paper and concentrated under reduced pressure; the concentrate was precipitated at $4^{\circ} \mathrm{C}$ for $24 \mathrm{~h}$ and then the precipitate was dried. Hops $(200 \mathrm{~g})$ were extracted twice with $70 \%$ ethanol $\left(85^{\circ} \mathrm{C}\right.$ and $4 \mathrm{~h}$ reflux). The extract was filtered through a filter paper, concentrated under reduced pressure, and then dried. Extracts were mixed at a weight ratio of $3: 1$ and used to assess in vivo efficacy.

2.2. High-Performance Liquid Chromatography (HPLC) Analysis of RHEC. An Agilent HPLC 1200 series system equipped with a quaternary pump, autosampler, and photodiode array detector with a Phenomenex ${ }^{\circledR}$ Luna C18 column $(250 \times 4.6 \mathrm{~mm}, 5 \mu \mathrm{m})$ was used for analysis (Phenomenex Inc., Torrance, CA, USA). Elution with solvent A (acetonitrile) and solvent B ( $0.25 \%$ formic acid in water) in a gradient elution at a flow rate of $1 \mathrm{~mL} / \mathrm{min}$ was carried out as follows: $0-3 \mathrm{~min}, 75 \% \mathrm{~B}$; 3-33 $\mathrm{min}, 75 \%-30 \% \mathrm{~B}$; 33-40 $\mathrm{min}$, $30 \%-10 \% \mathrm{~B}$; $40-45 \mathrm{~min}, 10 \% \mathrm{~B}$; $45-45.5 \mathrm{~min}, 10 \%-75 \% \mathrm{~B}$; and $45.5-50 \mathrm{~min}, 75 \% \mathrm{~B}$. The detection wavelength was set at $320 \mathrm{~nm}$. The column temperature was kept at $30^{\circ} \mathrm{C}$ and the injection volume was $10 \mu \mathrm{L}$.

2.3. Animals and Treatment. The study was approved by the Institutional Animal Care and Use Committee of Naturetech, Inc. (approval no. NT 11803), based on the National Animal Welfare Law of the Republic of Korea. Thirty-five virgin female Sprague-Dawley rats (7 weeks old) were obtained from OrientBio Co., Ltd. (Seongnam-si, South Korea). They were acclimated to the laboratory conditions for a week and housed in a specific pathogen-free room at a standard temperature of $24.0^{\circ} \mathrm{C} \pm 1.0^{\circ} \mathrm{C}$ with a humidity of $50 \% \pm 10 \%$ under a $12 \mathrm{~h}$ light/dark cycle. Water and feed
(ENVIGO 2018s, Envigo, Madison, WI, USA) were given ad libitum. One week after arrival, the rats were anesthetized using pentobarbital sodium $(40 \mathrm{mg} / \mathrm{kg})$ intraperitoneally and subjected to a bilateral ovariectomy (OVX) or sham operation via double dorsolateral incision. The experimental groups were divided into the following five groups ( 7 rats per group): Sham and OVX and RHEC 125, 250, and $500 \mathrm{mg} / \mathrm{kg}$ groups. At 4-5 days after surgery, ovariectomized animals received red clover and hops extract combination (RHEC 125,250 , and $500 \mathrm{mg} / \mathrm{kg}$, p.o.) and vehicle (distilled water) for 12 weeks. The body weights of all animals were examined at the beginning of the experiment and at weekly intervals throughout the 12-week experimental period. BW gain was calculated as the difference between initial and final body weights. Food consumption was recorded during the 12week period of RHED administration on a weekly basis and the mean daily food consumption was calculated using the total. At the end of the experiment, rats were anesthetized with $2 \%-3 \%$ isoflurane and blood samples were collected from the ventral aorta. The uterus, liver, kidney, and fat pads (visceral and retroperitoneal) were dissected out and weighed. Uteri were fixed in $10 \%$ buffered formaldehyde and blocked in paraffin. The paraffin block was cut into 3-5 $\mathrm{m}$ sections and stained with hematoxylin and eosin for light microscopic analysis.

2.4. Measurement of TST. On the day of measuring TST, vehicle or RHEC was administered orally $30 \mathrm{~min}$ before the measurements.

2.4.1. Under Natural Conditions. The rats were removed from the cage and placed on an experimental table. Then, only the tail was lightly held, and the temperature of the dorsal part of the tail about $2 \mathrm{~cm}$ away from the base was measured using an infrared thermometer (Model 153IRB, Bioseb, Chaville, France). After a total of three measurements, the average value was obtained.

2.4.2. Under Stressed Conditions. TST under stressed conditions was measured using a slightly modified method as previously described [15]. Rats were restrained in a holder in a conscious condition and TST was measured for $1 \mathrm{~h}$ on the dorsal side of the tail about $2 \mathrm{~cm}$ from the fur line using an infrared thermometer. Before testing, all animals were allowed to acclimate to the laboratory for $15 \mathrm{~min}$. The ambient temperature was $25^{\circ} \mathrm{C}$. TST data were measured at 10 min intervals throughout the experimental period.

2.5. Serum Analysis. Blood samples were centrifuged at $3,000 \mathrm{rpm}$ and $4^{\circ} \mathrm{C}$ for $15 \mathrm{~min}$; serum samples were collected and stored at $-70^{\circ} \mathrm{C}$ until analysis. Alanine aminotransferase (ALT), aspartate aminotransferase (AST), alkaline phosphatase (ALP), total cholesterol (T-Chol), triglyceride (TG), low-density-lipoprotein cholesterol (LDL-C), and highdensity-lipoprotein cholesterol (HDL-C) were measured using an automatic blood chemistry analyzer (BS-220, Mindray, Xuzhou City, China). Measurements of 
osteocalcin, C-telopeptide of type I collagen (CTX-1), superoxide dismutase (SOD), malonyl dehydrogenase (MDA), endothelin-1, and nitric oxide in serum were performed using the Rat Osteocalcin ELISA kit (Cusabio Biotech, Houston, TX, USA), RatLaps (CTX-1) EIA Assay (Immunodiagnostic System, Gaithersburg, MD, USA), OxiSelect ${ }^{\mathrm{TM}}$ Superoxide Dismutase Activity (Cell Biolabs, Inc., San Diego, CA, USA), OxiSelect ${ }^{\mathrm{TM}}$ TBARS (Thiobarbituric Acid Reactive Substances) Assay Kit (MDA Quantitation, Cell Biolabs Inc., San Diego, CA, USA), Quantikine ELISA Endothelin-1 kit (R\&D Systems, Minneapolis, USA), and colorimetric NO assay kit (R\&D Systems, Minneapolis, MN, USA), following the manufacturer's instructions. All samples were analyzed in duplicate in the assay.

2.6. Statistical Analysis. All statistical analyses were performed by one-way ANOVA using SAS software (SAS Institute Inc., Cary, NC, USA). The repeated measure was used for statistical analysis of the levels of body weight and TST over time. Multiple comparisons were performed with Duncan's multiple-range tests and Tukey-Kramer adjustment. Results are presented as mean \pm SD. Values of $P<0.05$ were considered to represent statistical significance.

\section{Results}

3.1. HPLC Analysis of RHEC. Based on the absorption and retention time of standard materials, biochanin $\mathrm{A}$ and xanthohumol (Figure 1(a)) were identified as major components of RHEC, and the results confirmed that RHEC contained $10.3 \pm 0.9 \mathrm{mg} / \mathrm{g}$ biochanin A (25.4 min) and $0.3 \pm 0.04 \mathrm{mg} / \mathrm{g}$ xanthohumol (38.1 min) (Figure 1(b)).

3.2. Body and Fat Weights. The changes of mean body weight throughout the experimental period are reported in Figure 2. The OVX group showed significantly increased weight change compared with the sham group. Oral administration of RHEC to ovariectomized rats for 12 weeks was observed to inhibit body weight gain during the entire experimental period. After 8 weeks of administration of RHEC at $500 \mathrm{mg} / \mathrm{kg}$, there was significant inhibition of the increase in body weight. In addition, body weight gain was significantly reduced at the end of the experiment in all treatment groups. Furthermore, after RHCE administration, the visceral and retroperitoneal fat weights were significantly decreased when compared with that in the OVX group. Feed intake in the OVX group was significantly higher than in the sham group but significantly decreased in the $120 \mathrm{mg} / \mathrm{kg}$ RHEC group. The 250 and $500 \mathrm{mg} / \mathrm{kg}$ RHEC groups also slightly decreased, but no significance was observed (Table 1).

3.3. Tail Skin Temperature (TST). Measurements of TST under natural conditions in weeks 4,8 , and 12 are shown in Figure 3(a), and detailed changes in TST on Week 4 are shown in Figure 3(b). The OVX group showed significant increases throughout the experiment compared with the sham group. The increase in TST by ovariectomy was markedly inhibited by the administration of RTEC. In addition, under the stress conditions, the OVX group showed significantly increased TST at 20-60 min after measurement (Figure 3(c)). However, TST in RHEC rats $(125,250$, or $500 \mathrm{mg} / \mathrm{kg})$ was significantly $(P<0.05)$ lower than that of the OVX group. In addition, regarding the change of TST during $60 \mathrm{~min}$, the temperature of the OVX group was significantly more elevated than that of the sham group and the RHEC groups showed a decreased range of TST in a dose-dependent manner (Figure 3(d)).

3.4. Lipid Profile. The OVX group showed significant elevations of total cholesterol, HDL-C, and LDL-C. After 12 weeks of administration of RHEC, total cholesterol significantly decreased in the RHEC $500 \mathrm{mg} / \mathrm{kg}$ group. Triglyceride also decreased in all treatment groups, albeit not significantly (Table 2 ).

3.5. Bone Metabolism-Related Biomarkers. Serum ALP, osteocalcin, and CTX-1 were significantly increased in the OVX group. However, in the RHEC-treated groups, these markers showed patterns of decreases in a dose-dependent manner; CTX-1 in particular was significantly decreased in the three dose groups (Table 2).

3.6. Antioxidant Capacity. The OVX group showed a decrease in SOD level in serum and an increase in MDA level when compared with the sham group (Table 2). However, the administration of RHEC at $500 \mathrm{mg} / \mathrm{kg}$ for 12 weeks significantly restored these levels.

3.7. Change in Vasorelaxation Markers. In the OVX group, plasma endothelin-1 was significantly increased when compared with the level in the sham group (Figure 4(a)), but nitric oxide was decreased (Figure 4(b)). However, these changes were significantly normalized by the administration of RHEC and showed a dose-dependent result (Figure 4).

3.8. Safety Evaluation of RHEC. After 12 weeks of administration of RHEC, organ weights of the liver and kidney (Table 1) and serum levels of ALT and AST (Table 2) did not show any differences. OVX rats showed the atrophy of endometrial epithelial cells, and RHEC groups showed the same microscopic findings as that of the OVX group (Table 1 and Figure 5); there were also no differences in uterus weights among the OVX and RHEC groups (Table 1).

\section{Discussion}

The estrogenic component found in plants is called phytoestrogen, which is present at an especially high level in Fabaceae. It exhibits activity similar to that of estrogen and is mostly a flavonoid and phenolic acid substance [16]. One of the most studied phytoestrogens is isoflavone. The isoflavone component of red clover binds to the estrogen receptor and is reported to significantly alleviate estradiol 


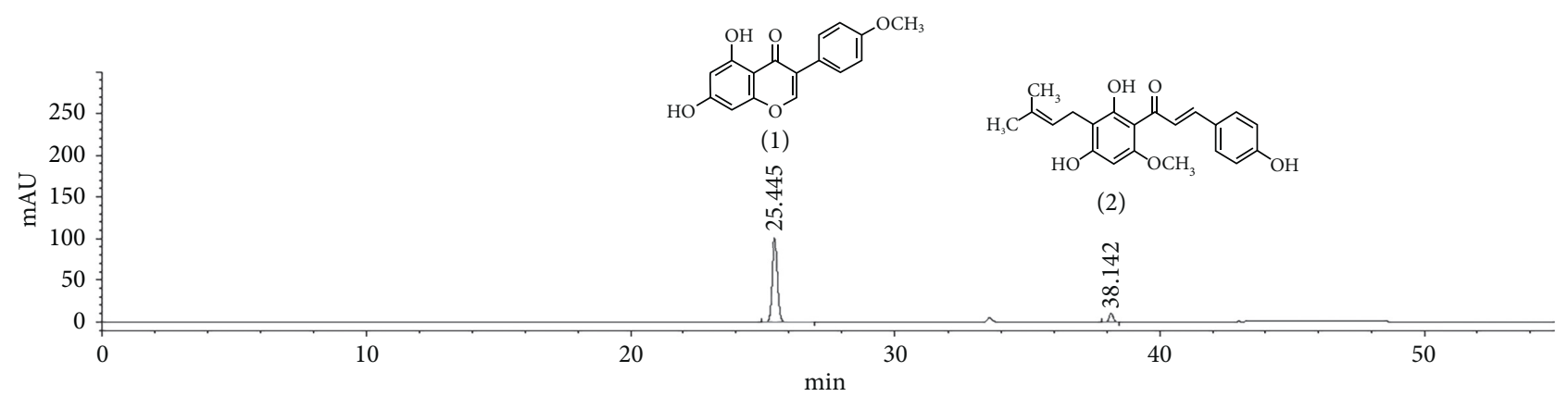

(a)

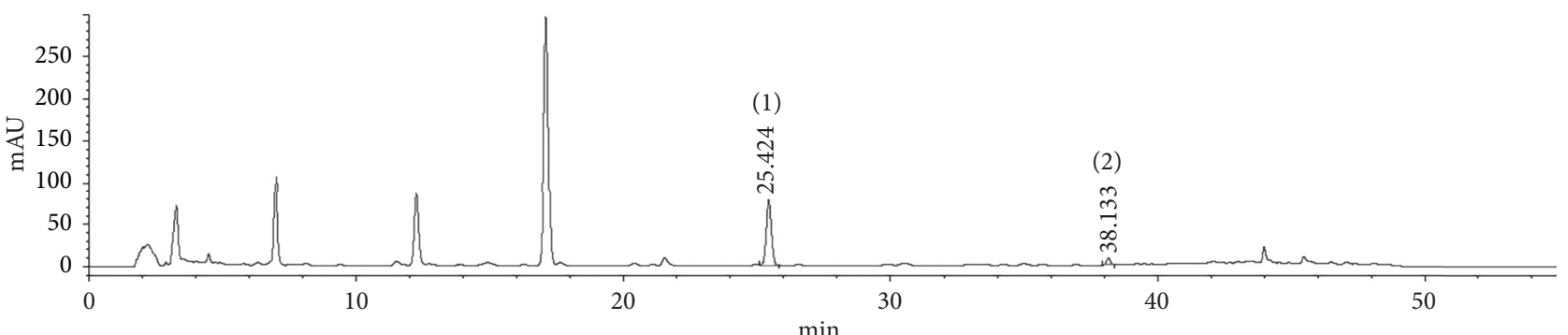

(b)

FIgURE 1: Representative HPLC chromatogram at $320 \mathrm{~nm}$ : standard solution (a) of biochanin A (1) and xanthohumol (2) and RHEC (b).

reduction associated with menopause when ingested with red clover [17, 18]. Xanthohumol, isoxanthohumol, and 8prenylnaringenin (8-PN) in hops are also known as phytoestrogens [19]. Red clover and hops extracts have been reported to improve the fragmentary symptoms of menopause, such as osteoporosis and hot flashes; however, their effectiveness is still being debated [20-22]. Building on this previous work, the present study was conducted to study the overall improvement of women's menopausal symptoms, such as accumulation of body fat, hot flashes, abnormal blood lipids, osteoporosis, and risk of CVD, by the administration of RHEC in OVX rats.

The synergistic effect of RHEC on the estrogen receptor was confirmed in our previous study (data in progress-under submission), and in this study, we confirmed its improvement of various symptoms associated with estrogen deficiency using an ovariectomized rat model.

Similar to a report describing that the reduction in estrogen secretion by ovarian resection causes weight gain [23], the body and fat weights of the rats in the OVX group were higher than that in the sham group. RHEC administration inhibited weight gain and abdominal fat accumulation, and this effect is similar to that seen after estrogen treatment in OVX rats [24]. This weight loss was not observed in the red clover alone [25]; weight loss was observed in high contents of xanthohumol in hops $[26,27]$. Biochanin $A$ and xanthohumol contents in RHEC were approximately $1.09 \%$ and $0.033 \%$, respectively. Although RHEC showed similar or lower contents than the active compounds mentioned in previous studies $[25,26]$, it showed weight gain inhibition and abdominal fat accumulation effects. Because we have

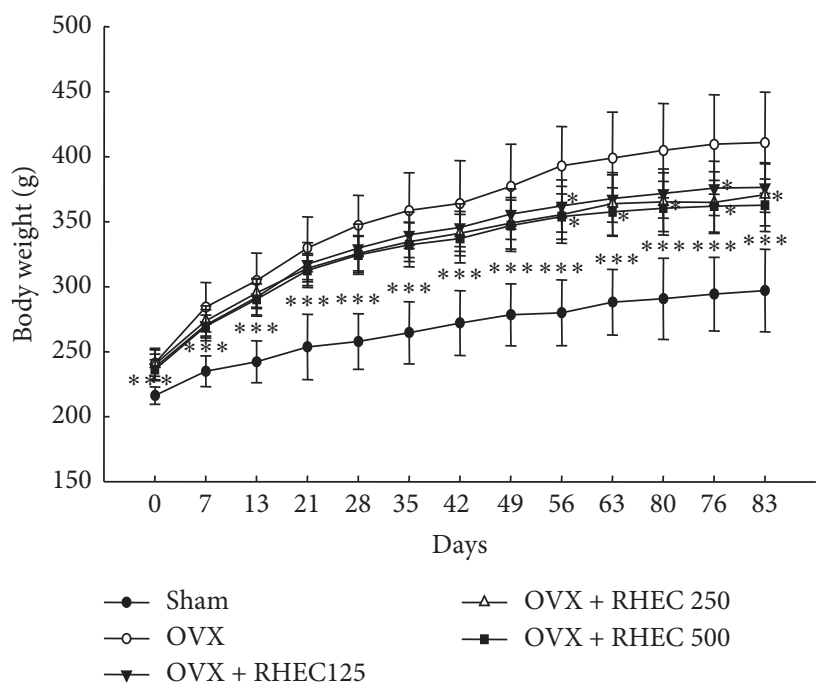

FIgUre 2: Changes in body weight during 12 weeks of RHEG administration. Values are presented as mean $\pm \mathrm{SD}(n=7)$. ${ }^{*} p<0.05,{ }^{* *} p<0.01$, and ${ }^{* * *} p<0.001$ versus OVX.

already confirmed the effects of estrogen receptor activity on RHEC through in vitro tests (data in progress-under submission), this effect is expected to be via estrogen receptor activity.

One of the main symptoms of menopausal women, hot flashes, is a temporary symptom involving severe fever of the neck and face, which causes sweating and chills and greatly reduces the quality of life. The precise mechanism behind hot flashes is unclear, but they are speculated to be caused by 
TABLE 1: Biometric changes after 12 weeks of RHEC administration to OVX rats.

\begin{tabular}{|c|c|c|c|c|c|}
\hline Group & Sham & OVX & RHEC (125) & RHEC (250) & RHEC (500) \\
\hline Body weight gain (g) & $80.74 \pm 28.43^{c}$ & $169.50 \pm 32.26$ & $138.30 \pm 12.9^{\mathrm{a}}$ & $130.31 \pm 20.76^{\mathrm{a}}$ & $126.53 \pm 17.56^{\mathrm{a}}$ \\
\hline Visceral fat $(\mathrm{g})$ & $3.42 \pm 1.82^{\mathrm{c}}$ & $7.84 \pm 1.40$ & $5.73 \pm 1.53$ & $5.21 \pm 1.41^{\mathrm{a}}$ & $4.74 \pm 0.74^{\mathrm{b}}$ \\
\hline Retroperitoneal fat (g) & $3.23 \pm 1.39^{c}$ & $8.16 \pm 2.17$ & $6.29 \pm 1.88$ & $5.78 \pm 0.82^{\mathrm{a}}$ & $5.27 \pm 0.58^{\mathrm{c}}$ \\
\hline Liver $(\mathrm{g})$ & $6.75 \pm 0.87$ & $7.69 \pm 0.66$ & $6.75 \pm 0.37$ & $6.96 \pm 0.73$ & $6.67 \pm 0.67$ \\
\hline Kidney (g) & $1.64 \pm 0.14$ & $1.68 \pm 0.09$ & $1.64 \pm 0.19$ & $1.60 \pm 0.07$ & $1.58 \pm 0.19$ \\
\hline Uterus $(\mathrm{g})$ & $0.54 \pm 0.06^{\mathrm{a}}$ & $0.11 \pm 0.02$ & $0.09 \pm 0.01$ & $0.11 \pm 0.02$ & $0.11 \pm 0.01$ \\
\hline Uteric epithelial height $(\mu \mathrm{m})$ & $27.71 \pm 5.36^{\mathrm{a}}$ & $14.34 \pm 2.78$ & $15.12 \pm 1.97$ & $14.30 \pm 2.63$ & $14.97 \pm 2.59$ \\
\hline Feed intake (g/day) & $16.47 \pm 1.07^{\mathrm{c}}$ & $19.98 \pm 1.17$ & $18.23 \pm 0.89^{\mathrm{a}}$ & $18.61 \pm 0.75$ & $18.38 \pm 1.25$ \\
\hline
\end{tabular}

Values are expressed as mean $\pm \mathrm{SD}(n=7)$. OVX, ovariectomy; RHEC, combination of red clover extract and hops extract; FER, food efficiency ratio. ${ }^{a} P<0.05$ versus OVX; ${ }^{\mathrm{b}} P<0.01$ versus OVX; ${ }^{\mathrm{c}} P<0.001$ versus OVX.

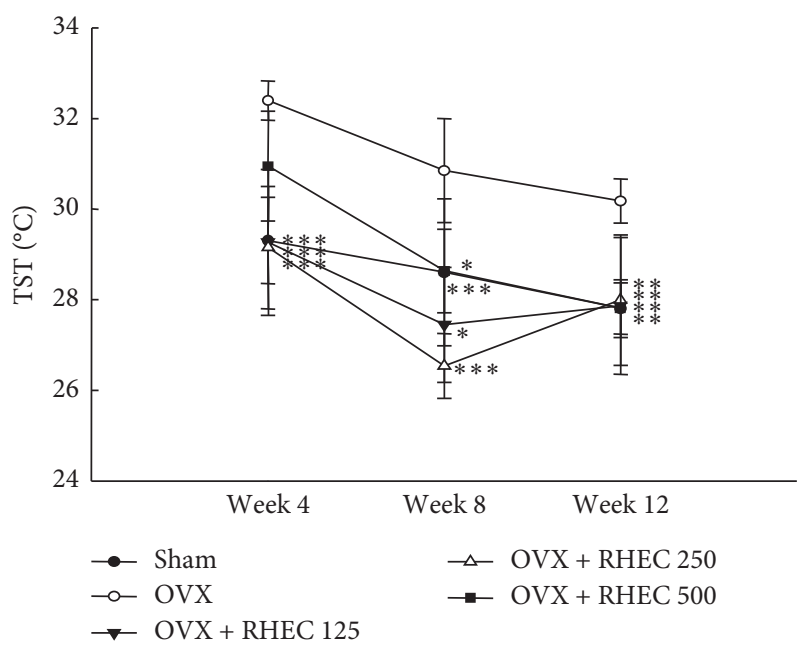

(a)

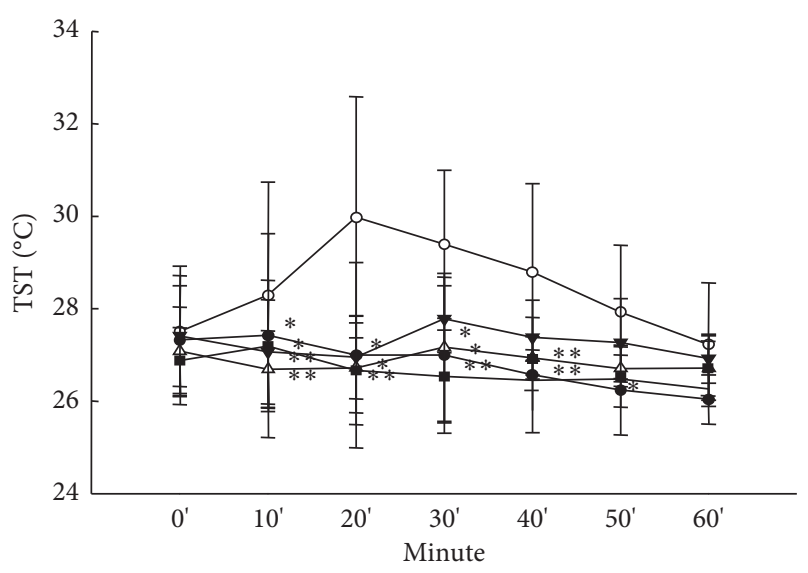

(c)

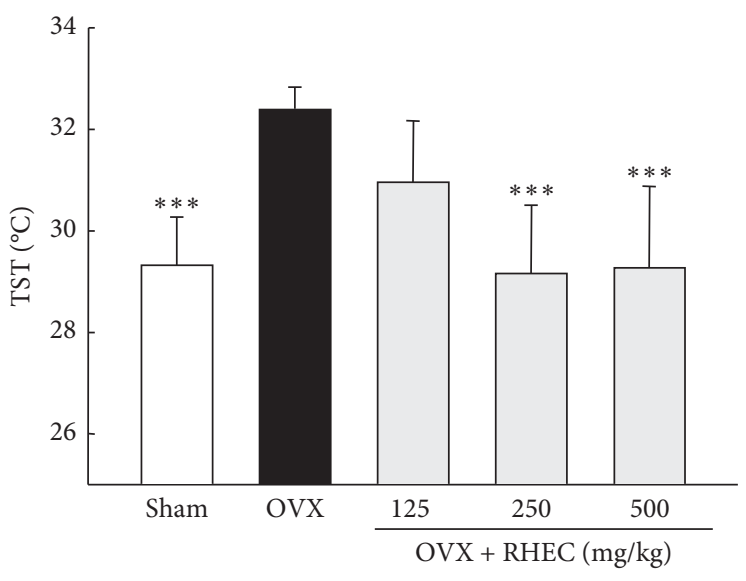

(b)

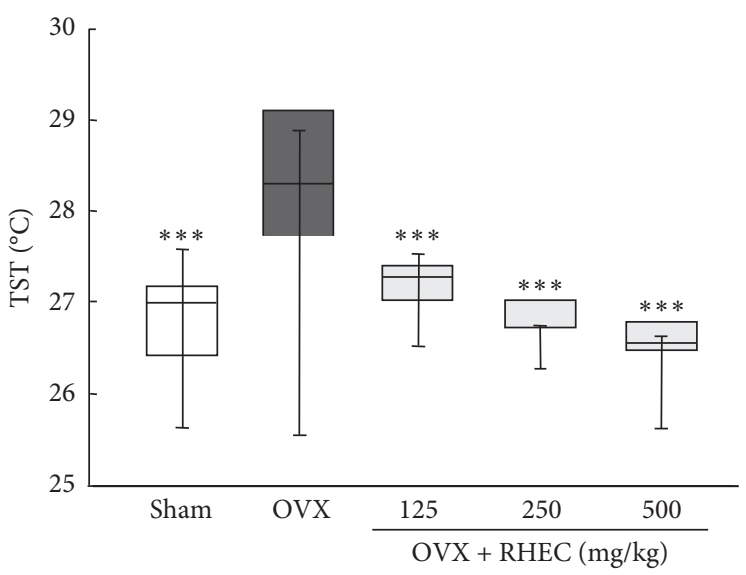

(d)

FIGURE 3: Results of tail skin temperature (TST) measured under natural conditions from weeks 4 to 12 (a) and TST in Week 4 (b). TST under the stress conditions for $1 \mathrm{~h}$ (c) and temperature range during $1 \mathrm{~h}(\mathrm{~d})$. Values are presented as mean $\pm \mathrm{SD}(n=7)$. ${ }^{*} p<0.05,{ }^{* *} p<0.01$, and ${ }^{* * *} p<0.001$ versus OVX.

dysfunction of the autonomic nervous system caused by rapid heat loss due to increased peripheral blood flow and skin conductivity [28]. In addition, estrogen is considered to play an important role in hot flashes because they appear during menopause, when blood levels of estrogen are low [29]. TST was measured in this experiment. In the RHEC group, an increase in TST was significantly suppressed under normal as well as under stress conditions. It was confirmed that TST decreased when 8-PN, one of the main components of hops, was administered to OVX rats [30]; moreover, it was found that changes in TST and rectal temperature decreased upon consumption of Lifenol ${ }^{\circledR}$ [27], a standardized hops $\mathrm{CO}_{2}$ extract. While the clinical test results of red clover for the iris have been abundantly reported [31], there are few 
TABLE 2: Results of serum analysis after 12 weeks of RHEC administration to OVX rats.

\begin{tabular}{|c|c|c|c|c|c|}
\hline \multirow{2}{*}{ Group } & \multirow{2}{*}{ Sham } & \multirow{2}{*}{ OVX } & \multicolumn{3}{|c|}{ OVX } \\
\hline & & & RHEC (125) & RHEC (250) & RHEC (500) \\
\hline $\operatorname{ALT}(\mathrm{U} / \mathrm{L})$ & $35.8 \pm 10.6$ & $37.0 \pm 2.5$ & $38.8 \pm 5.9$ & $36.9 \pm 4.1$ & $35.3 \pm 2.9$ \\
\hline AST (U/L) & $72.5 \pm 17.1$ & $78.0 \pm 5.6$ & $85.9 \pm 8.3$ & $79.0 \pm 8.3$ & $72.9 \pm 4.9$ \\
\hline T-Chol (mg/dL) & $76.0 \pm 14.7^{\mathrm{c}}$ & $123.7 \pm 17.6$ & $117.1 \pm 14.1$ & $109.6 \pm 9.0$ & $93.6 \pm 10.4^{c}$ \\
\hline $\mathrm{TG}(\mathrm{mg} / \mathrm{dL})$ & $69.1 \pm 16.4$ & $100.4 \pm 38.7$ & $78.0 \pm 23.8$ & $73.1 \pm 10.2$ & $65.7 \pm 14.5$ \\
\hline HDL-C (mg/dL) & $58.5 \pm 10.6^{\mathrm{a}}$ & $73.0 \pm 6.1$ & $81.2 \pm 8.8$ & $79.4 \pm 6.3$ & $67.7 \pm 7.4$ \\
\hline LDL-C (mg/dL) & $6.8 \pm 109^{c}$ & $13.0 \pm 1.8$ & $15.2 \pm 2.4$ & $13.6 \pm 1.2$ & $11.4 \pm 1.1$ \\
\hline $\operatorname{ALP}(\mathrm{U} / \mathrm{L})$ & $41.1 \pm 10.6^{c}$ & $69.7 \pm 9.8$ & $74.0 \pm 17.6$ & $58.1 \pm 11.2$ & $52.9 \pm 5.1$ \\
\hline Osteocalcin $(\mathrm{pg} / \mathrm{ml})$ & $771.4 \pm 322.9^{\mathrm{b}}$ & $1723.3 \pm 153.6$ & $1743.3 \pm 425.5$ & $1498.1 \pm 267.8$ & $1265.1 \pm 127.9$ \\
\hline CTX-1 $(\mathrm{pg} / \mathrm{ml})$ & $7.15 \pm 1.64^{c}$ & $15.40 \pm 3.90$ & $11.81 \pm 2.63^{\mathrm{a}}$ & $11.53 \pm 1.30^{\mathrm{b}}$ & $6.48 \pm 2.02^{\mathrm{c}}$ \\
\hline $\mathrm{SOD}(\mathrm{U} / \mathrm{ml})$ & $42.625 \pm 12.856^{\mathrm{a}}$ & $17.423 \pm 14.266$ & $37.263 \pm 22.755$ & $46.711 \pm 11.368^{\mathrm{a}}$ & $48.704 \pm 14.952^{\mathrm{b}}$ \\
\hline $\operatorname{MDA}(\mu \mathrm{M} / \mathrm{ml})$ & $10.24 \pm 3.45^{\mathrm{b}}$ & $19.22 \pm 3.86$ & $14.65 \pm 5.19$ & $13.74 \pm 3.50$ & $11.54 \pm 4.61^{\mathrm{a}}$ \\
\hline
\end{tabular}

Values are expressed as mean \pm SD $(n=7)$. OVX, ovariectomy; RHEC, combination of red clover extract and hops extract. ${ }^{\mathrm{a}} P<0.05$ versus $\mathrm{OVX}$; ${ }^{\mathrm{b}} P<0.01$ versus OVX; ${ }^{c} P<0.001$ versus OVX.

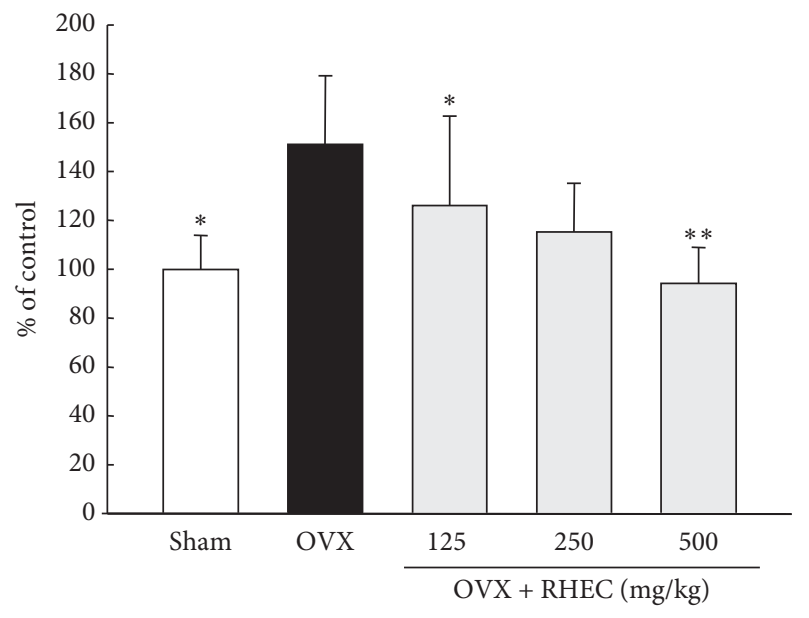

(a)

FIgURE 4: Results of endothelin-1 (a) and nitric oxide ${ }^{*} p<0.05,{ }^{* *} p<0.01$, and ${ }^{* * *} p<0.001$ versus OVX.

reports about TST results in animals. In this study, we found that the TST range of fluctuation by physical stress decreased in the RHEC group, suggesting that the interaction of RE and $\mathrm{HE}$ may affect central thermoneutral and peripheral vasodilation.

Estrogen deficiency is involved in the development of cardiovascular disease (CVD) in women, and it plays a role in endothelial function and vascular tone, among others [32]. Newson [33] recommends early and active prevention and response for managing cardiovascular risk factors. Therefore, the prevention of cardiovascular risk factors is considered one of the main indicators when evaluating the efficacy of RHEC. In this study, the levels of TC, TG, HDL-C, and LDL-C related to cardiovascular disease were measured and the levels of ET-1 and NO were analyzed in the blood to evaluate endothelial function. The results showed significant reductions in T-Chol in the RHEC $500 \mathrm{mg} / \mathrm{kg}$ dose group, along with tendencies for reduction in the levels of TG and LDL-C. These results are similar to those observed in dyslipidemia due to estrogen reduction being alleviated by estrogen replacement therapy [34]. Furthermore, RE and HE

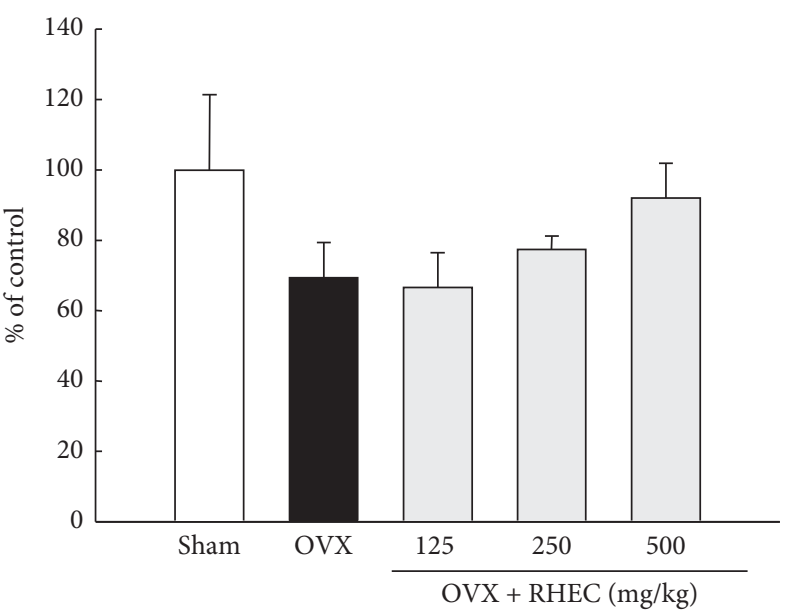

(b)

(b) in serum. Values are presented as mean $\pm \mathrm{SD} \quad(n=7)$.

have been observed to have efficacy related to lipid metabolism. A clinical study reported that TC and LDL-C levels decreased during the 3-month intake of RE [35] and that HDL-C levels increased after 1 year of administration [36]. Many studies have also reported about a link between menopausal conditions and elevated blood lipid levels [37].

Estrogens play an important role in skeletal homeostasis, and hormonal deficiency in the ovaries is one of the greatest risk factors of osteoporosis [17]. The ingestion of estrogen has been reported to have an effect on the prevention of bone fractures by increasing the level of inorganic matter in bone and the composition of collagenous fibers [38]. Estrogen is also known to affect bone resorption [18]. In the OVX group, significant increases in blood osteocalcin and blood ALP levels, which are active indicators of osteoblasts, were observed compared with those in the sham group; furthermore, significant decreases in osteocalcin and ALP levels were observed in the RHEC dose group. RE increases BMD, bone minerals, and cancellous bone mass, indicating that red clover affects bone metabolism $[17,25,39]$. It also appears to reduce bone loss by reducing 


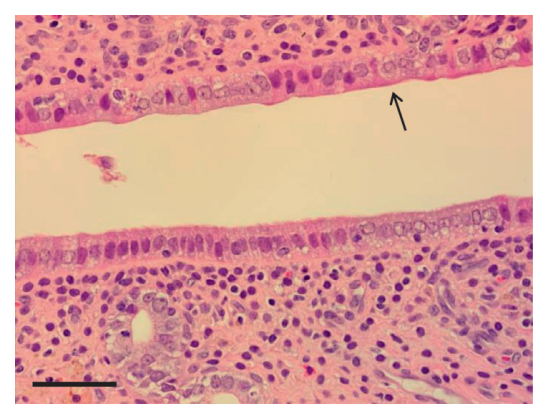

(a)

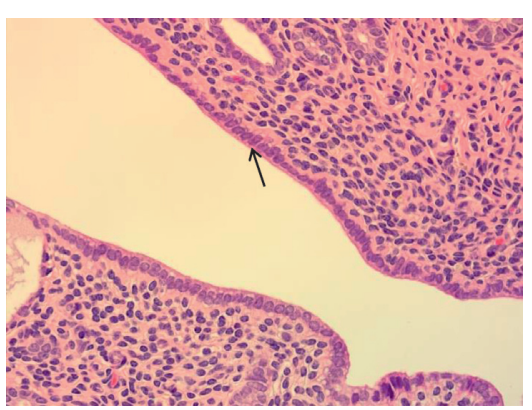

(d)

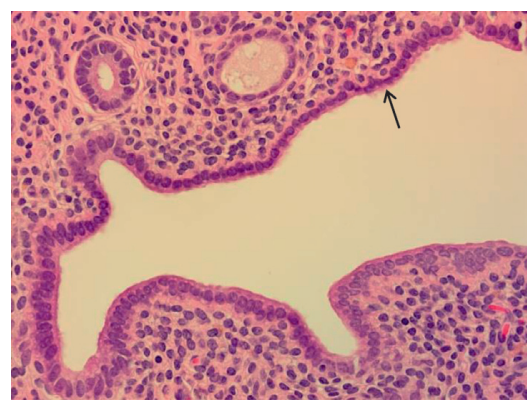

(b)

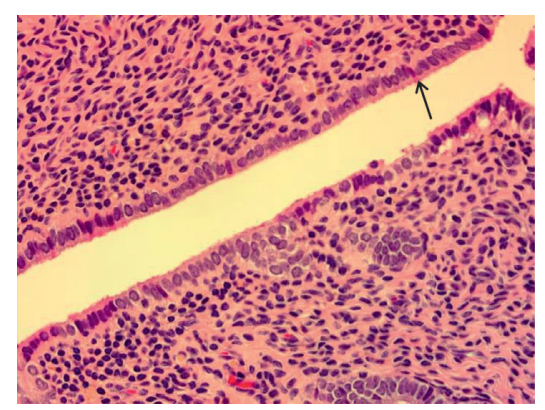

(c)

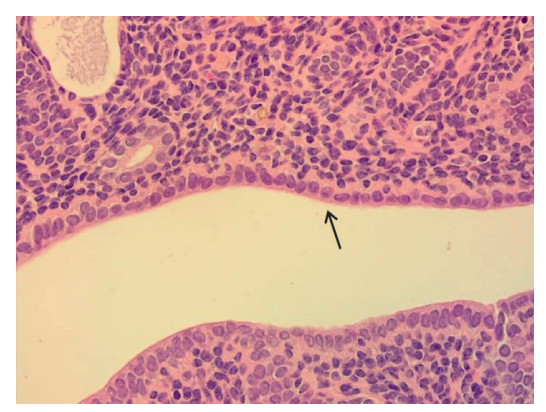

(e)

Figure 5: H\&E staining of the uterus. Sham (a), OVX (b), RHEC $125 \mathrm{mg} / \mathrm{kg}$ (c), RHEC $250 \mathrm{mg} / \mathrm{kg}$ (d), and RHEC $500 \mathrm{mg} / \mathrm{kg}$ (e). Black arrows indicate the epithelial layer of the endometrium. The bar indicates $50 \mu \mathrm{m}$ (magnification $\times 200)$.

bone turnover by inhibiting ALP and bone resorption [40]. HE has also been reported to prevent the reduction of trabecular thickness due to estradiol deficiency and to reduce the number of osteoclasts in the tibial metaphysic [41]. CTX-1 is a bone resorption marker produced by the activity of osteoclasts [42]. Our findings show that the $3: 1$ complex inhibits bone resorption. It was confirmed that blood ALP, osteocalcin, and CTX-1 were reduced in the RHEC group, suggesting that RHEC could improve bonerelated symptoms.

Decreased estrogen levels in blood increase antioxidant stress [43], and these changes are associated with increased climacteric symptoms and increased CVD risk [44]. In this experiment, it was confirmed that oxidative stress was reduced by the administration of RHEC. Antioxidant activity due to red clover and HE has been reported as one of the mechanisms by which RHEC administration alleviates menopausal symptoms $[45,46]$.

An increase in endothelin- 1 and a decrease in NO are representative symptoms of endothelial dysfunction, which are also observed in estrogen-deficient women and ovariectomized animals. Estrogen therapy can protect against this imbalance in endothelial function and diminish the risk of CVD $[47,48]$. In the present study, endothelial function was normalized by RHEC administration similar to the case of estrogen administration; it is thus expected to have an effect of reducing the risk of CVD following menopause. Moreover, we identified a synergistic effect of the components of RHEC using HUVECs.

Hormone therapy (HT) used to be the standard of care for managing vasomotor symptoms (VMS) and for preventing chronic diseases until the publication of the
Women's Health Initiative in 2002. At present, HT is only approved for four conditions [43]: VMS management, menopausal bone loss, and vulvovaginal and genitourinary symptoms in women $<60$ years old who are within 10 years of menopause and have no contraindications. Otherwise, the use of HT for the primary prevention of other chronic conditions such as CHD, breast cancer, and dementia remains controversial [44]. The estrogen hormone and estrogenic photochemical usually act via estrogen receptors (ER). These have shown agonistic action with high binding affinity for ER $\alpha$ (highly expressed in cancer-sensitive tissues) [49] and ER $\beta$; isoflavones retain selective affinity for ER $\beta$, which is present in tissues requiring certain stimulation by estrogen to function normally (e.g., bone tissue, bone marrow, adipose, brain, kidney, endothelial cells, and liver) [50]. The binding affinity to ER of RHEC was tested and it was seen that RHEC had more selective affinity to ER $\beta$ (affinity ratio $\mathrm{ER} \alpha: \mathrm{ER} \beta=1: 1.2$ ). Therefore, when studying phytoestrogen or other estrogenic phytochemicals, in addition to the positive effects of estrogen, there is also a need to assess the risks of proliferation and bleeding of uterine tissue and to demonstrate safety. In this study, it was determined that RHEC had no histological effects on uterine tissues when OVX endometrial tissues were administered it for 12 weeks. Therefore, RHEC can act as an alternative to the currently used HT in improving women's menopausal symptoms.

\section{Conclusion}

In conclusion, RHEC appears to have high potential for comprehensively improving various symptoms of 
menopause. Further studies on its human application and mechanism of action are needed. Furthermore, more investigation is needed on how $\mathrm{RE}$ and $\mathrm{HE}$ interact with the inhibition of fat accumulation and the relief of parasympathetic nervous system symptoms improve the lipid profile and correct bone metabolism. Nonetheless, it is expected to be useful as a new alternative for treating menopause.

\section{Data Availability}

Our data are from the analysis of articles and can be provided when necessary.

\section{Conflicts of Interest}

The authors declare that they have no conflicts of interest.

\section{Authors' Contributions}

Mi Ran Kim was responsible for methodology, investigation, formal analysis, and writing the original draft. Hyun Jin Kim was responsible for methodology, investigation, data curation, formal analysis, writing, reviewing, and editing. Su Hyun $\mathrm{Yu}$ was performed investigation, data curation, and formal analysis. Bo Su Lee was responsible for investigation and resource. Se Yeong Jeon performed formal analysis and investigation. Jeong Jun Lee was responsible for supervision and project administration. Young Chul Lee was responsible for conceptualization, methodology, data curation, writing, reviewing, and editing.

\section{Acknowledgments}

This research was financially supported by the Ministry of SMEs and Startups (MSS), Korea, under the "Regional Specialized Industry Development Program (R\&D, P0003019)" supervised by the Korea Institute for Advancement of Technology (KIAT).

\section{References}

[1] M. R. Soules, S. Sherman, E. Parrott et al., "Executive summary: stages of reproductive aging workshop (STRAW)," Fertility and Sterility, vol. 15, no. 1, pp. 1-5, 2001.

[2] M. Sussman, J. Trocio, C. Best et al., "Prevalence of menopausal symptoms among mid-life women: findings from electronic medical records," BMC Womens. Health, vol. 15, no. 1, pp. 1-5, 2013.

[3] G. K. Poomalar, "The quality of life during and after menopause among rural women," Journal of Clinical and Diagnostic Research, vol. 7, no. 7, pp. 135-139, 2013.

[4] V. Beck, U. Rohr, and A. Jungbauer, "Phytoestrogens derived from red clover: an alternative to estrogen replacement therapy?" The Journal of Steroid Biochemistry and Molecular Biology, vol. 94, no. 5, pp. 499-518, 2005.

[5] A. C. Thorup, M. N. Lambert, H. S. Kahr, M. Bjerre, and P. B. Jeppesen, "Intake of novel red clover supplementation for 12 weeks improves bone status in healthy menopausal women," Evidence-Based Complementary and Alternative Medicine, vol. 2015, Article ID 689138, 11 pages, 2015.
[6] C. Atkinson, W. Oosthuizen, S. Scollen, A. Loktionov, N. E. Day, and S. A. Bingham, "Modest protective effects of isoflavones from a red clover-derived dietary supplement on cardiovascular disease risk factors in perimenopausal women, and evidence of an interaction with ApoE genotype in 49-65 Year-old women," The Journal of Nutrition, vol. 134, no. 7, pp. 1759-1764, 2004.

[7] M. P. Warren and S. Halpert, "Hormone replacement therapy: controversies, pros and cons," Best Practice \& Research Clinical Endocrinology \& Metabolism, vol. 18, no. 3, pp. 317-332, 2004.

[8] J. E. Rossouw, G. L. Anderson, R. L. Prentice et al., "Risks and benefits of estrogen plus progestin in healthy postmenopausal women: principal results from the women's health initiative randomizes controlled trial," JAMA, vol. 288, no. 3, pp. 321-333, 2002.

[9] D. F. P. Mainini G, M. Torella, M. C. Di Donna et al., "Nonhormonal management of postmenopausal women: effects of a red clover based isoflavones supplementation on climacteric syndrome and cardiovascular risk serum profile," Clinical and Experimental Obstetrics and Gynecology, vol. 40, no. 3, pp. 337-341, 2013.

[10] S.-M. Heinonen, K. Wähälä, and H. Adlercreutz, "Identification of urinary metabolites of the red clover isoflavones formononetin and biochanin A in human subjects," Journal of Agricultural and Food Chemistry, vol. 52, no. 22, pp. 6802-6809, 2004.

[11] P. Zanoli and M. Zavatti, "Pharmacognostic and pharmacological profile of Humulus lupulus L," Journal of Ethnopharmacology, vol. 116, no. 3, pp. 383-396, 2008.

[12] C. R. Overk, P. Yao, L. R. Chadwick et al., "Comparison of the in vitro estrogenic activities of compounds from hops (Humulus lupulus) and red clover (Trifolium pratense)," Journal of Agricultural and Food Chemistry, vol. 53, no. 16, pp. 6246-6253, 2005.

[13] A. Constituents, "Humulus Lupus. Monograph." Alternative Medicine Review, vol. 8, no. 2, pp. 190-192, 2003.

[14] A. K. Zołnierczyk, W. K. Mączka, M. Grabarczyk et al., "Isoxanthohumol - biologically active hop flavonoid," Fitoterapia, vol. 103, pp. 71-82, 2015.

[15] G. Lee, J. Kim, H. Kang et al., "Effects of herbal mixture extract on menopausal hot flashes and pharmacokinetics in ovariectomized rat models," Journal of Food and Nutrition Research, vol. 6, no. 2, pp. 116-123, 2018.

[16] K. Yazaki, K. Sasaki, and Y. Tsurumaru, "Prenylation of aromatic compounds, a key diversification of plant secondary metabolites," Phytochemistry, vol. 70, no. 15-16, pp. 17391745, 2009.

[17] S. Kawakita, F. Marotta, Y. Naito et al., "Effect of an isoflavonescontaining red clover preparation and alkaline supplementation on bone metabolism in ovariectomized rats," Clinical Interventions in Aging, vol. 4, no. 1, pp. 91-100, 2009.

[18] K. M. Prestwood, C. C. Pilbeam, J. A. Burleson et al., "The short-term effects of conjugated estrogen on bone turnover in older women," The Journal of Clinical Endocrinology \& Metabolism, vol. 79, no. 2, pp. 366-371, 1994.

[19] S. R. Milligan, J. C. Kalita, A. Heyerick, H. Rong, L. De Cooman, and D. De Keukeleire, "Identification of a potent phytoestrogen in hops (Humulus lupulus L.) and beer," The Journal of Clinical Endocrinology \& Metabolism, vol. 84, no. 6, p. 2249, 1999.

[20] R. Kargozar, H. Azizi, and R. Salari, "A review of effective herbal medicines in controlling menopausal symptoms," Electronic Physician, vol. 9, no. 11, pp. 5826-5833, 2017. 
[21] J. Kolodziejczyk-Czepas, "Trifoliumspecies - the latest findings on chemical profile, ethnomedicinal use and pharmacological properties," Journal of Pharmacy and Pharmacology, vol. 68, no. 7, pp. 845-861, 2016.

[22] A. Keiler, O. Zierau, and G. Kretzschmar, "Hop extracts and hop substances in treatment of menopausal complaints," Planta Medica, vol. 79, no. 07, pp. 576-579, 2013.

[23] J. R. Bracht, V. J. Vieira-potter, R. D. S. Santos et al., "The role of estrogens in the adipose tissue milieu," Annals of the New York Academy of Sciences, vol. 1461, pp. 1-17, 2019.

[24] R. Meli, M. Pacilio, G. M. Raso et al., "Estrogen and raloxifene modulate leptin and its receptor in hypothalamus and adipose tissue from ovariectomized rats," Endocrinology, vol. 145, no. 7, pp. 3115-3121, 2004.

[25] U. Cegieła, J. Folwarczna, M. Pytlik, and G. Zgórka, "Effects of extracts from Trifolium medium L. and Trifolium pratense L. on development of estrogen deficiency-induced osteoporosis in rats," Evidence-Based Complementary and Alternative Medicine, vol. 2012, Article ID 921684, 11 pages, 2012.

[26] K. Yui, A. Kiyofuji, and K. Osada, "Effects of xanthohumolrich extract from the hop on fatty acid metabolism in rats fed a high-fat diet," Journal of Oleo Science, vol. 63, no. 2, pp. 159-168, 2014.

[27] Y. H. Ban, J. M. Yon, Y. Cha et al., "A hop extract lifenol ${ }^{\circledR}$ improves postmenopausal overweight, osteoporosis, and hot flash in ovariectomized rats," Evidence-based Complementary and Alternative Medicine, vol. 2018, Article ID 2929107, 9 pages, 2018.

[28] F. Kronenberg, L. J. Cote, D. M. Linkie, I. Dyrenfurth, and J. A. Downey, "Menopausal hot flashes: thermoregulatory, cardiovascular, and circulating catecholamine and $\mathrm{LH}$ changes," Maturitas, vol. 6, no. 1, pp. 31-43, 1984.

[29] H. H. G. Berendsen, A. H. J. Weekers, and H. J. Kloosterboer, "Effect of tibolone and raloxifene on the tail temperature of oestrogen-deficient rats," European Journal of Pharmacology, vol. 419, no. 1, pp. 47-54, 2001.

[30] J. Bowe, X. F. Li, J. Kinsey-Jones et al., "The hop phytoestrogen, 8-prenylnaringenin, reverses the ovariectomy-induced rise in skin temperature in an animal model of menopausal hot flushes," Journal of Endocrinology, vol. 191, no. 2, pp. 399-405, 2006.

[31] M. Ghazanfarpour, R. Sadeghi, R. L. Roudsari, I. Khorsand, T. Khadivzadeh, and B. Muoio, "Red clover for treatment of hot flashes and menopausal symptoms: a systematic review and meta-analysis," Journal of Obstetrics and Gynaecology, vol. 36, no. 3, pp. 301-311, 2016.

[32] M. E. Mendelsohn and R. H. Karas, "Molecular and cellular basis of cardiovascular gender differences," Science, vol. 308, no. 5728, pp. 1583-1587, 2005.

[33] L. Newson, "Menopause and cardiovascular disease," Post Reproductive Health, vol. 24, no. 1, pp. 44-49, 2018.

[34] R. K. Ross, A. Paganini-Hill, T. M. Mack, and B. E. Henderson, "Cardiovascular benefits of estrogen replacement therapy," American Journal of Obstetrics and Gynecology, vol. 160, no. 5, pp. 1301-1306, 1989.

[35] P. Chedraui, G. San Miguel, L. Hidalgo, N. Morocho, and S. Ross, "Effect of Trifolium pratense-derived isoflavones on the lipid profile of postmenopausal women with increased body mass index," Gynecological Endocrinology, vol. 24, no. 11, pp. 620-624, 2008.

[36] M. M. Terzic, J. Dotlic, S. Maricic, T. Mihailovic, and B. TosicRace, "Influence of red clover-derived isoflavones on serum lipid profile in postmenopausal women," Journal of Obstetrics and Gynaecology Research, vol. 35, no. 6, pp. 1091-1095, 2009.
[37] H.-S. Kim, "Menopause-associated lipid metabolic disorders and foods beneficial for postmenopausal women," Nutrients, vol. 12, no. 1, p. E202, 2020.

[38] A. P. Clark and J. A. Schuttinga, "Targeted estrogen/progestogen replacement therapy for osteoporosis: calculation of health care cost savings," Osteoporosis International, vol. 2, no. 4, pp. 195-200, 1992.

[39] S. Kang, B. Choi, S. Kim et al., "Dried pomegranate potentiates anti-osteoporotic and anti-obesity activities of red clover dry extracts in ovariectomized rats," Nutrients, vol. 7, no. 4, pp. 2622-2647, 2015.

[40] F. Occhiuto, R. D. Pasquale, G. Guglielmo et al., "Effects of phytoestrogenic isoflavones from red clover (Trifolium pratense L.) on experimental osteoporosis," Phytotherapy Research, vol. 21, no. 2, pp. 130-134, 2007.

[41] A. M. Keiler, J. Helle, M. I. Bader et al., "A standardized Humulus lupulus (L.) ethanol extract partially prevents ovariectomy-induced bone loss in the rat without induction of adverse effects in the uterus," Phytomedicine, vol. 34, pp. 50-58, 2017.

[42] Y.-S. Lee, R. Gupta, J.-T. Kwon et al., "Effect of a bisphosphonate and selective estrogen receptor modulator on bone remodeling in streptozotocin-induced diabetes and ovariectomized rat model," The Spine Journal, vol. 18, no. 10, pp. 1877-1887, 2018.

[43] F. Bellanti, M. Matteo, T. Rollo et al., "Sex hormones modulate circulating antioxidant enzymes: impact of estrogen therapy," Redox Biology, vol. 1, no. 1, pp. 340-346, 2013.

[44] A. Cagnacci, M. Cannoletta, F. Palma, M. Bellafronte, C. Romani, and B. Palmieri, "Relation between oxidative stress and climacteric symptoms in early postmenopausal women," Climacteric, vol. 18, no. 4, pp. 631-636, 2015.

[45] H. Mu, Y.-H. Bai, S.-T. Wang, Z.-M. Zhu, and Y.-W. Zhang, "Research on antioxidant effects and estrogenic effect of formononetin from Trifolium pratense (red clover)," Phytomedicine, vol. 16, no. 4, pp. 314-319, 2009.

[46] A. Di Sotto, P. Checconi, I. Celestino et al., "Antiviral and antioxidant activity of a hydroalcoholic extract from humulus lupulus L." Oxidative Medicine and Cellular Longevity, vol. 2018, Article ID 5919237, 14 pages, 2018.

[47] F. Moien-Afshari, E. Kenyon, J. C. Choy, B. Battistini, B. M. McManus, and I. Laher, "Long-term effects of ovariectomy and estrogen replacement treatment on endothelial function in mature rats," Maturitas, vol. 45, no. 3, pp. 213223, 2003.

[48] Z. Tan, T.-H. Wang, D. Yang, X.-D. Fu, and J.-Y. Pan, "Mechanisms of $17 \beta$-estradiol on the production of ET- 1 in ovariectomized rats," Life Sciences, vol. 73, no. 21, pp. 2665-2674, 2003.

[49] W. Yue, J. D. Yager, J.-P. Wang, E. R. Jupe, and R. J. Santen, "Estrogen receptor-dependent and independent mechanisms of breast cancer carcinogenesis," Steroids, vol. 78, no. 2, pp. 161-170, 2013.

[50] N. Heldring, A. Pike, S. Andersson et al., "Estrogen receptors: how do they signal and what are their targets," Physiological Reviews, vol. 87, no. 3, pp. 905-931, 2007. 